\title{
La enseñanza del inglés en escuelas primarias santafesinas en territorios de pobreza: pensar una perspectiva crítica en didáctica específica. Contribuciones desde un estudio de tesis de maestría
}

Palabras clave. saberes socialmente productivos . construcción de subjetividad · lengua extranjera . perspectiva crítica $\cdot$ pobreza

Resumen. El artículo presenta parte de las conclusiones de una tesis de maestría que estudia el trabajo con una lengua extranjera en escuelas primarias en contextos de vulnerabilidad de la ciudad de Santa Fe. Retoma una prescripción que se deriva de la Ley de Educación Nacional 26206/06 en cuyo capítulo II, artículo 87, dice: «La enseñanza de al menos un idioma extranjero será obligatoria en todas las escuelas primarias y secundarias del país». Así, la tesis estudia el aula de 7mo. grado, en la que el inglés es un espacio curricular, de escuelas enclavadas en barrios marginales de la ciudad, y analiza condiciones institucionales y pedagógicas necesarias para su implementación desde una perspectiva didáctica crítica. Se trata de un trabajo donde pobreza y enseñanza

(1) Maestra. Profesora de Inglés. Licenciada en Inglés. Maestra de grado en Escuela Primaria No Graduada de Avance Continuo Gral. Pascual Echagüe. Profesora de Inglés en Escuela Secundaria ${ }^{\circ} 2020$ San Isidro Labrador, en Santa
Rosa de Calchines y Directora Escuela Secundaria Orientada $N^{\circ}$ 5II Juana Azurduy. Docente en ISP $\mathrm{N}^{\circ} 8$ en las cátedras Seminario de Niños en Riesgo Social y Problemáticas Contemporáneas de la Educación Primaria in y en

SANDRA TREVISANI ${ }^{(1)}$

se van constituyendo en territorios con particularidades que los identifican y diferencian, en los que se visibiliza a niños sujetos de cultura y en los que se va planteando la impronta de una práctica docente como proceso no enajenante, con un discurso no hegemónico que valore lo cultural propiciando la construcción de identidad y subjetividad de estos tiempos. De este modo, se estudian las posibilidades de construcción de saberes socialmente productivos en el aula. El reconocimiento de las condiciones con que cuenta la escuela primaria santafesina hoy abona la posibilidad de construir ciudadanías críticas para empoderar a las infancias de los territorios santafesinos pobres y habilitar emancipaciones, con la convicción de que puedan fortalecer su ciudadanía.

ISP $\mathrm{N}^{\circ}$ IO en Inglés Técnico. Integrante de Equipo de Capacitación en Educación de Adultos dependiente de la Dirección Provincial de Educación de Adultos e integrante de la Comisión redactora del Diseño
Curricular de Educación Primaria de la Educación de Jóvenes y Adultos en Lengua Extranjera. Dictado de cursos sobre Alfabetización y TIC en ISP $\mathrm{N}^{\circ}$ IO aprobados por el Infod. trevisanisandra@gmail.com 


\section{English teaching in primary schools of areas affected \\ by poverty in Santa F: thinking a critical perspective in specific didactics. Contributions from a study for a master's degree thesis}

\begin{abstract}
The present article presents a part of a master's thesis conclusion that studies teaching a foreign language (English) in primary schools in contexts of vulnerability in Santa Fe, Argentina. It takes over a prescription derived from the national education act (Ley Nacional de Educación Nr. 26206/06), whose Article 87, Chapter II, reads: The teaching of at least one foreign language will be compulsory in all primary and secondary schools in the country. This thesis studies the 7th grade class -where English is a curricular subject- of schools located in marginal neighborhoods of the city and analyzes the necessary institutional and pedagogical conditions for its implementation from the point of view of critical didactics. Poverty and education constitute territories with particularities that identify and differentiate them; in which children, as culture sub-
\end{abstract}

jects, are made visible, and in which a teaching practice is considered as a non-alienating process with a non-hegemonic discourse that consider cultural aspects promoting the construction of identity and subjectivity. In this way, the possibilities of building socially productive knowledge in the classroom are studied. The recognition of primary schools' available conditions today in Santa Fe enables the possibility of building critical citizenship to empower children in poor territories and allow them to emancipate under the conviction that they can strengthen their citizenship.

Keywords. esocially productive knowledge . construction of subjectivity · foreign language · critical perspective $\cdot$ poverty 
(1) En adelante haré mención a la expresión «en los bordes» para referirme a sectores ubicados al margen de la ciudad y a la orilla del río

Salado. Pretendo dar cuenta de una ubicación geográfica que conlleva, en sí, una lógica de vida y una mirada social de los sujetos que los habitan.

(2) Proyecto de ley presentado por el diputado Carlos Comi a la Honorable Cámara de Diputados de la Nación, ( $E l$ Parlamentario, Io/03/20II) Disponible en: http:// www.parlamentario.com/ noticia-34577.html

\section{Inquietudes iniciales}

Luego de más de dos décadas de docencia en los bordes ${ }^{(1)}$ de la ciudad de Santa $\mathrm{Fe}$, hay cuestiones centradas en las prácticas de la enseñanza del inglés que me preocupan y me ocupan, más aún luego de que se declarara la obligatoriedad de la enseñanza de una lengua extranjera para el nivel primario en la Ley de Educación Nacional 26206/16, capítulo II, artículo 87, el que prescribe: «La enseñanza de al menos un idioma extranjero será obligatoria en todas las escuelas primarias y secundarias del país». Además, según expresa un proyecto de ley presentado a la Honorable Cámara de Diputados de la Nación, existe la posibilidad de que esta lengua extranjera sea el inglés. ${ }^{(2)}$

Mi preocupación por la enseñanza de esta lengua en los bordes se funda en lo inédito del abordaje del idioma desde los primeros grados del nivel primario, ya que, a partir de la implementación de la Ley Federal de Educación en la provincia de Santa Fe, en 1999, la escuela primaria pública comenzó a enseñar inglés como lengua extranjera en $7 \mathrm{mo}$. grado (aun cuando estaba prevista desde 4to.), proceso que se continúa desarrollando en la actualidad. Con excepción de las escuelas normales superiores, que tienen régimen diferente y cuentan con la oferta del idioma desde 1er. grado, no existe la oferta de inglés dentro de la currícula desde los primeros años del resto de las escuelas primarias públicas, ni en las escuelas privadas que funcionan en los barrios de los márgenes de Santa Fe. Solo las escuelas privadas del casco céntrico cuentan con esta oferta curricular.

La idea de un diálogo entre el territorio y una perspectiva crítica en la Didáctica de la Lengua Extranjera constituyó la inquietud inicial para desarrollar un estudio que conforma una tesis de maestría titulada «La enseñanza del inglés en la escuela primaria: desafíos para pensar una didáctica específica en contextos de pobreza». Me propuse indagar en los significados asignados a la enseñanza del inglés por los diferentes actores institucionales; identificar aquellos rasgos constitutivos de las prácticas de enseñanza del inglés en la escuela primaria que aporten al campo de una didáctica específica crítica de la lengua extranjera en contextos de vulnerabilidad; analizar las condi- 
ciones pedagógicas e institucionales que posibilitarían la implementación de la obligatoriedad del inglés como Lengua Extranjera desde los primeros años de la escuela primaria en contextos de pobreza; y aportar al análisis de las funciones sustantivas de la escuela en la construcción de subjetividades.

Se realiza una exploración desde el territorio, desde las experiencias cotidianas en las escuelas para reconocer, desde allí, las particularidades de la enseñanza de esta lengua extranjera en escuelas signadas por el desamparo y las múltiples pobrezas (Sirvent, 1996). La vinculación entre los sujetos que las habitan interroga las posibilidades que estas tienen de construir subjetividades con las nuevas infancias así como tornan visibles los puntos de contacto entre la lengua extranjera, el papel del entorno y, obviamente, la construcción de su propia identidad.

¿Cuál es el lugar de la escuela en este proceso y la importancia de los aprendizajes situados en los bordes de Santa Fe hoy, signados por la inmediatez y la incertidumbre, imbuidos en un escenario complejo en el que se construyen nuevas identidades? ¿Qué sentido tiene la enseñanza del inglés en estas experiencias? Dar algunas respuestas también implica plantear estrategias que posibiliten la formación de ciudadanos críticos, considerar las condiciones con que cuenta la escuela para diseñar y recrear dispositivos pedagógicos y políticas institucionales.

Entiendo que atender estos interrogantes nos anima a advertir configuraciones pedagógicas e institucionales que enriquecen una didáctica desde una perspectiva crítica que dialoga con el contexto, ofrece nuevos sentidos al trabajo de enseñanza de una lengua extranjera en los bordes santafesinos, y fortalece la construcción de identidades y el desarrollo de un proyecto emancipador.

En este escrito presento algunas de estas consideraciones que son las que conforman el corpus teórico de la tesis y sostienen la naturaleza del trabajo empírico que acompañó y enriqueció sustancialmente el análisis y, finalmente, comparto parte de las correspondientes conclusiones. 


\section{Pobrezas, márgenes y escuelas}

Pensar la escuela desde su inscripción en el territorio supone percibirla desde la construcción del vínculo simbólico que la une con aquellos que la habitan. La experiencia escolar está signada por tensiones que pugnan entre continuidades y rupturas, pero sobre todo por un estilo atravesado por fuertes lazos de implicación con el lugar ocupado, no como simple territorio sino como aquello que construye reconocimiento. Duschatzky (2008) afirma que entrar a la escuela es abrir una cadena de significantes, es unirse a un universo que nombra a los jóvenes como sujetos portadores de derechos. La escuela que trabaja con la cultura de y en la periferia, la de los saberes experienciales, no siempre autorizados, es la que porta soportes y lenguajes que no siempre participan de la cotidianeidad de los sectores populares, pero sí de un imaginario con el que quisiera fundirse. Sus ritmos y horarios quiebran la inercia de lo habitual. Reconocer el contexto de la escuela y las condiciones de vida de niños y adultos, implica poder comprender las relaciones de desigualdad ocultas bajo la enunciación del concepto de «diversidad» y cambiar la lente por la que se mira, indignarse al entender los procesos socioeconómicos que generan esta situación y también concebirla como un espacio de resistencia, pensado como un «lenguaje de la posibilidad» (Giroux, 1997).

\section{La enseñanza del inglés y los territorios de pobreza}

La perspectiva sobre la enseñanza de un idioma extranjero en territorios de pobreza, en este trabajo, apuesta a una acción pedagógica que no enajene ni instale un discurso hegemónico que quiebre la autonomía o debilite la construcción de identidad, naturalizando las diferencias. Enseñar inglés en los márgenes es un desafío que, lejos de ser nuevo, al menos en el nivel primario, se viene dando desde la implementación de la Ley Federal de Educación en los 7 mos. años de las escuelas de toda la provincia de Santa Fe. La cuestión central de este desafío está en descubrir los intersticios, los pliegues, en donde reside la relación particular que tienen niños y niñas que habitan los bordes urbanos de nuestra ciudad con una lengua que está en permanente contacto con su lengua materna. En palabras de Zárate: 
Adoptando estrechamente sus prácticas de enseñanza al contexto local, el docente confirma la tecnicidad de su profesión, no solo dominando la lengua que enseña, sus referencias y su campo disciplinar, sino también analizando la relación particular entre el entorno cultural de su enseñanza y la lengua y la cultura que enseña. (1995:71, en Manga, 2008)

Hablar de la enseñanza del inglés hoy implica, indudablemente, trascender el escenario local. Una mirada sobre el contexto mundial nos permite observar una realidad con rasgos complejos: el fenómeno de la globalización tanto en lo económico como en lo político y cultural. Gutiérrez (2010) cataloga de innegable la expansión de la lengua inglesa a lo largo y a lo ancho del globo y por lo tanto advierte dos cuestiones importantes: por un lado, la amenaza que ésta impone a la diversidad lingüística y califica a este fenómeno como «depredador», y, por otro, el reconocimiento explícito del acceso libre a la comunicación a todos los ciudadanos. La primera adhiere a la idea de imperialismo cultural y la segunda responde a una visión amplia en la que se van desdibujando los límites lingüísticos para dar lugar a la comunicación sin barreras. En este momento vivimos en un territorio global, Internet nos dispuso en un espacio digital en el cual la lengua que circula casi con preponderancia es el inglés.

¿Cómo se va construyendo la práctica docente en una sociedad plagada de contrastes donde conviven la pobreza y el desempleo junto con Facebook, Yahoo, WhatsApp, Instagram, Google, entre otros dispositivos supuestamente pensados para agilizar las comunicaciones? Creo que se trata, además, de sumar a esta práctica docente un modo de transitar las nuevas infancias y adolescencias, con nuevas ciudadanías e identidades, nuevos espacios comunicacionales por medio de los cuales se superan las fronteras espaciales y territoriales y se abre la posibilidad de «andar» otros mundos. Evidentemente, asumirnos como ciudadanos de «estos tiempos» implica asumir cambios tanto en lo tecnológico como en lo comunicacional, una nueva percepción de lo cercano y lo lejano, de nosotros y los «otros». Me refiero a cambios y complejidades que nos remiten a paradigmas complejos, donde se integran miradas para construir nuevas subjetividades vinculadas con lo nuevo y que advierten los cambios producidos en la realidad que vivimos hoy.

Asumirse docente de una lengua extranjera en el escenario social actual supone abordar el conocimiento y su enseñanza desde una perspectiva donde los 
problemas de las sociedades complejas trascienden la clásica separación de disciplinas para su abordaje. Podría pensarse aquí al lenguaje como punto de encuentro entre lo social e individual, lengua y habla, discurso entendido como texto y contexto. Sagastizabal afirma que «el lenguaje es un hecho social en sí y que vincula al individuo con la sociedad, lo particular con lo universal, al presente con el pasado, a la esfera biológica con la antropo-social» (2006:34). Convendría comenzar a pensar qué se está haciendo y qué podría hacerse dentro de un espacio donde el sujeto que enseña y el que aprende están involucrados en esta realidad dinámica, compleja, incierta. En palabras de Brailovsky:

[s]e analiza entonces la propia posibilidad de educar en el contexto de una escuela escindida entre grandes afanes y profundos cuestionamientos. En la sociedad del «just do it» y el «impossible is nothing», se propone reflexionar acerca de algunos puntos de imposibilidad de la educación que son, además, puntos de partida para lo posible. (2008:23)

Presentaré dos dimensiones que configuran una perspectiva crítica en Didáctica que entiendo vertebradoras para pensar la enseñanza del inglés en contextos de pobreza. Hago referencia a: a) la de los aprendizajes y saberes y b) la de la práctica docente.

\section{a) Aprendizajes vinculados con lo social y cultural}

La construcción del papel social y cultural del conocimiento, para esta investigación, es fundamental en términos de vinculación de los sujetos con la vida ciudadana y el ejercicio de sus derechos, es por ello que se aborda la categoría saberes socialmente productivos (Puiggrós, 2004). Se trata de saberes del sujeto que transforman la naturaleza y la cultura, enriqueciendo el capital cultural y de los que la escuela no puede estar ajena porque está inmersa en entornos en los que estos circulan. La tarea docente está en contribuir a la reconstitución de una trama social dañada, desde la enseñanza de una lengua ofrecer nuevos sentidos para regenerar vínculos sociales. En esta perspectiva, el contacto con las comunidades, con los grupos y las personas, sus experiencias, constituyen la oportunidad de instituir nuevos sentidos y asignarle valor pedagógico a eso 
que llamamos saberes, pero no a los saberes aislados de los contextos y de las necesidades sociales sino aquellos que hoy son capaces de integrar e incluir socialmente a nuestros alumnos, de construir y reconstruir su cultura en espacios que signifiquen su reconocimiento.

¿Podríamos hablar de la lengua extranjera como uno de los elementos que hay que comenzar a incluir como parte de la reconstrucción de una trama social que hoy nos atraviesa?

Una de las responsabilidades de la educación y más precisamente la escuela actual es contribuir en la construcción del tejido social dañado que nos dejaron las diferentes crisis por las que atravesó nuestro país (dictadura militar, neoliberalismo de los años 90, crisis de 2001, entre otras). También es tarea de la escuela identificar cuáles son aquellos saberes que construyen y precisan construir nuestros niños y jóvenes hoy y que hay que comenzar a incluir en esta nueva trama social y en el currículo escolar. Aquí se encuentra la lengua extranjera, desafiando las nuevas subjetividades, las nuevas infancias, poniendo en tensión los saberes heredados con aquellos que hoy surgen como necesidad de un contacto permanente con un mundo lejano y que podemos apreciar y vivenciar a través de múltiples formatos.

La construcción de identidad no puede pensarse sin la experiencia, en cuanto a su legado histórico cultural, sus formas de vida y trabajo presentes, y en cuanto a sus rasgos de subjetividad, también. La experiencia es compleja, involucra diversas dimensiones, tiempos e historias. A partir de estos principios podemos pensar en contenidos curriculares, no sólo centrados en contenidos de la ciencia, sino articulados a los contenidos de la experiencia. Siguiendo a Orozco (en Gómez Sollano, 2009), lo socialmente significativo nos invita necesariamente a pensar el aprendizaje como un intercambio y lucha de códigos culturales.

Al considerar a la Didáctica y su relación con los problemas de la actualidad, Klett (2013) propone una didáctica de las lenguas extranjeras —en plural-, una didáctica transversal. Destaca la posibilidad de que las lenguas extranjeras se conecten con otras ciencias con las que tienen mucho en común: psicología, ciencias sociales, ciencias del lenguaje y de la educación. Deja de lado la posibilidad de una didáctica única y monolítica para todas las lenguas extranjeras, a la vez que tampoco se proclama a favor de didácticas específicas de cada 
lengua aislada. Más bien propicia un diálogo plurilingüe configurado en una didáctica amplia y diversificada de las lenguas extranjeras, que está abierta a las modificaciones necesarias, que también genere la posibilidad de recibir y procesar las diferencias de cada lengua y de cada cultura.

Kumaravadivelu (2001) plantea un debate acerca del método y la necesidad de buscar opciones poco convencionales que permitan a los mismos docentes planificar y buscar su propia manera de enseñar sin seguir principios artificiales que en general están alejados del aula (Klett (2013). Kumaravadivelu (2001) propone la condición del posmétodo como la posibilidad de trascender las limitaciones del método y encontrar una alternativa para diseñar estrategias de enseñanza y estructurar los conocimientos acerca de la lengua (la lengua extranjera, en nuestro caso) y pretende revisar la relación entre teóricos y docentes pensando en estrategias que puedan implementarse en las aulas hoy, en las que el docente tenga autonomía y donde se relacionen teoría y práctica. Sostiene que se debe facilitar el avance de una enseñanza de idiomas contextualizada basada en una verdadera comprensión de particularidades lingüísticas, socioculturales y políticas locales. Obliga a repensar la relación entre teóricos y docentes permitiendo a los profesores construir su propia teoría de la práctica. Kumaravadivelu $(1994,2003)$ propone un marco estratégico que comprende diez macro-estrategias como un modo de hacer docencia e incorporar en la enseñanza de lenguas, nuevos parámetros. Se trata de pautas amplias que, según él, pueden orientar a los docentes en el diseño de sus propias estrategias, en sus propios contextos y con sus propias necesidades y se refieren a nociones sobre la enseñanza y el aprendizaje de una lengua extranjera: maximizar las oportunidades de aprendizaje, facilitar la interacción y la negociación, minimizar las confusiones de percepción de los alumnos y docentes, activar aprendizajes intuitivos, promover la conciencia acerca del lenguaje y contextualizar la información lingüística, integrar habilidades lingüísticas, promover la autonomía del alumno, desarrollar la conciencia cultural y garantizar la relevancia social de la enseñanza y el aprendizaje.

Cobran particular relevancia, para este trabajo, las macroestrategias relacionadas con el aprendizaje de la lengua extranjera, en tanto particularizado, contextualizado y con posibilidad de conocer el contexto para comprender las 
necesidades lingüísticas. Asimismo, las estrategias vinculadas a la conciencia cultural y la sensibilización con el entorno social, político, económico y educativo por cuanto permiten pensar en sujetos signados por una cultura, atravesados por fenómenos sociales y políticos que nos comprometen.

\section{b) La práctica docente: trabajo crítico y politicidad}

El proceso de construcción de la práctica docente, desde una perspectiva crítica en la Didáctica, requiere admitir a la crítica como sostenedora de las prácticas democráticas y al contexto como un lugar de preponderancia y sensibilidad, por cuanto es el lugar donde la política, el poder y la pedagogía se nutren mutuamente. Peter McLaren y Joe Kincheloe (2008) puntualizan que la teoría está representada por los modos de interpretación, análisis y generalizaciones acerca de cómo y por qué funciona el mundo, y el desarrollo de la consciencia crítica está relacionado con la comprensión, el análisis, la capacidad de preguntarse y de intervenir en la realidad social, cultural y económica de nuestra comunidad. Esta perspectiva advierte que no es posible escindir el trabajo del aula de las condiciones sociales, políticas y económicas que se presentan en la comunidad. En definitiva, se trata de prácticas democráticas en el aula, de poner en tensión todo lo relacionado con la representatividad (o no) de las minorías, el lugar de la comunidad pobre en un mundo globalizado latinoamericano, el papel de lo popular en la cultura de nuestros alumnos, las dimensiones hegemónicas y antihegemónicas de las nuevas tecnologías y de los medios de comunicación. Giroux reafirma que

[e]s imposible separar lo que hacemos en el aula de las condiciones políticas y económicas que determinan nuestro trabajo, y eso significa que la pedagogía debe ser entendida como una forma de trabajo académico en la que las cuestiones de tiempo, autonomía, libertad y poder son tan importantes para la clase como lo que se está enseñando. (en Gómez Sollano, 2009:20)

Silvana Barboni (2011), en su trabajo «Enseñanza de Inglés e Identidad Nacional. A los 200 años de la Revolución de Mayo», relaciona estas prácticas críticas con la enseñanza del inglés. Sostiene que esta lengua hoy nos instala en el foco 
de un debate postcolonial de resistencia y apropiación. Habla de una resistencia sutil pero poderosa y de una apropiación en términos de oportunidades de interacción para enriquecer la visión del mundo, de la propia realidad. Deja de lado la posibilidad de enseñar desde el vacío cultural.

\section{El trabajo con el territorio: decisiones metodológicas para aprender a mirar y a escuchar al otro}

Las dos escuelas con las que trabajé en esta investigación están estratégicamente ubicadas en ingresos a la ciudad, muy cerca de ríos, geografía característica de la ciudad de Santa Fe. Una se encuentra situada en el acceso sudoeste a la ciudad capital, en el barrio San Jerónimo (más conocido como FONAVI), dentro del barrio Centenario, que históricamente ha sido un barrio obrero, con altos índices de desocupación en la actualidad. Hoy se ve afectado por problemas de indigencia y marginalidad, se encuentra ubicado en el borde de la ciudad y rodeado, además, de asentamientos populosos a la orilla del río. Escuela del sudoeste para este trabajo.

La otra escuela se sitúa en barrio Alto Verde, ubicado al margen del acceso noreste a la ciudad, donde se encuentra el Puente Colgante, rodeada de ríos y lagunas, como mezclándose con clubes que interaccionan con el canotaje, con familias de pescadores, desocupados, y con serios problemas de violencia. Escuela de la costa para esta tesis.

Llevé a cabo este trabajo en sucesivas visitas acordadas con los actores que allí trabajan. La información obtenida fue recogida a través de entrevistas semiestructuradas realizadas a determinados docentes y directivos que trabajan en ellas, incluyendo datos y tópicos que consideré relevantes. Respecto de las preguntas que orientaron las entrevistas, procuraron indagar en las consideraciones y opiniones de los entrevistados acerca de lo que viven y sienten dentro del aula, la escuela y la comunidad, con el objeto de despertar la palabra y habilitar decires que dejen traslucir la relación entre el campo disciplinar y la enseñanza y la pobreza. En palabras de Carr y Kemmis (1988), episteme y tecné, entrelazándose para descubrir qué hay detrás de lo teórico, traspasando nuestra mera observación, lo que se nos muestra de lo real. También realicé 
observaciones y notas de campo que tomé en cada lugar, tratando de registrar lo que cada territorio erigía.

Así, los maestros de 7mo. grado, las profesoras de inglés de esos grupos y los vicedirectores de las escuelas seleccionadas, como principal fuente de información, son el puente para poder conocer los diferentes aspectos de la escuela y de la comunidad. El hecho de estar cara a cara con estos docentes me permitió poder abordar cuestiones no previstas y fue una ocasión significativa para que emergieran dimensiones «no pensadas» para considerar el problema. La libertad del entrevistado de elegir el momento y el lugar para el encuentro le otorgó al diálogo, sin dudas, un canon de importancia ya que ayudó a descubrir aquellos "rincones», sonidos y voces que hacen y hablan de la escuela.

Sautu (2003) especifica supuestos en esta metodología de análisis cualitativo: la aparición de pautas y rasgos estructurales que se exhiben en la conversación, la influencia del contexto en expresiones y la consideración de todos los detalles sin descartar nada a priori. Amparo Tusón Valls destaca «que el instrumento privilegiado que posee el ser humano para dar sentido a las diferentes circunstancias en que se encuentra es, precisamente, el uso lingüístico ya que es en la interacción verbal donde emergen los significados sociales» (2002:135)

Analizar sus palabras me posibilitó construir categorizaciones, reconocer conceptos, obtener información sobre el objeto de este trabajo: la enseñanza de la lengua extranjera en contextos de vulnerabilidad. El material de campo me posibilitó analizar la práctica docente, encontrar, en la palabra de los entrevistados, aquellas frases significativas, que me invitaban a reconocer múltiples consideraciones sobre el contexto, la escuela y los procesos de enseñanza y de aprendizajes que allí transcurren y devienen. En estas direcciones pude armar relaciones entre hechos, argumentos, categorías teóricas, identificar unos ejes estructurantes en cada contexto investigado, sostener no sin dificultad, la relación dinámica entre lo conceptual, lo descriptivo y los relatos.

Las implicancias de desarrollar este trabajo desde una perspectiva metodológica cualitativa requirieron una suerte de fuerte toma de distancia, una salida del juego que se juega cotidianamente — del que soy parte también como docente y como directivo de una escuela-, hacer desaparecer las urgencias, hacer discontinuo el tiempo continuo, ver en un instante hechos que se producen en 
una sucesión, hacer aparecer relaciones que en la acción son imperceptibles, algo así como romper el encanto.

Para vivenciar el contexto en el que se desarrolla la práctica, fue necesario recorrer el barrio, preguntar a los mismos chicos por algunas lógicas establecidas o instituidas, sentir aromas que los identifican, reconocer contrastes que le otorgan sentido. Sin dudas, estas vivencias, junto con la observación de los recreos y los diferentes momentos de la vida de la escuela, me permitieron una construcción aproximada de estos «lugares» en el sentido en que lo plantea Achilli (1990) cuando, en alusión a la perspectiva socioantropológica y su indagación de los problemas socioeducativos de nuestras sociedades complejas, afirma que se trata de un enfoque constructivista indiciario: un camino que se «va haciendo» sin estandarización alguna.

\section{A modo de corolario y de inicio, para seguir pensando}

Este trabajo de tesis procuró deconstruir la cotidianeidad de las prácticas escolares, de las prácticas de enseñanza del inglés en particular, advertir los supuestos y concepciones que las sostienen, así como reconocer sus perspectivas en torno al nivel y al contexto socio cultural. Es decir, comprender la trama social, histórica y política que constituye la enseñanza del inglés, la pobreza, la escuela. Así, se exploraron las configuraciones que constituyen estas prácticas para poder analizar la construcción de la enseñanza en una sociedad situada, estratégicamente, al margen del circuito laboral, del sistema educativo, de las oportunidades reales de acceso.

A través de este análisis, entonces, pude advertir que existen condiciones y dispositivos con los que cuenta la escuela primaria como institución formadora de identidades culturales, que posibilitarían la implementación de la obligatoriedad de la enseñanza del inglés como lengua extranjera para trabajar en contextos de pobreza en la ciudad de Santa Fe. Decido presentarlas en dos grandes grupos. En el primer grupo considero aquellas condiciones con las que ya cuenta la escuela y cuyo fortalecimiento contribuiría al enriquecimiento de las políticas institucionales. Hablo de condiciones institucionales que están instaladas y/o podrían optimizarse en la escuela primaria y que colaborarían para pensar 
rasgos que enriquecerían la enseñanza de la lengua extranjera, no solo en el espacio de séptimo sino pensando en la obligatoriedad del inglés desde primer grado. Así, identifico las siguientes: la regularidad de las reuniones plenarias; los espacios de planificación; el trabajo en equipo con maestros que tienen una fuerte presencia y carga horaria; el trabajo integrado con las áreas de especialidades; la supervisión y acompañamiento de un integrante del equipo directivo. Los espacios de planificación son generados por la institución, tienen cierta regularidad y se sostienen en el tiempo, poseen autorización y legitimidad institucional y se desarrollan durante las jornadas escolares. Allí se realizan acuerdos. Se trata de espacios de intercambio y debate de ideas donde los maestros, los profesores de las especialidades (salvo los profesores de inglés) y los integrantes de los equipos de gestión consensuan trabajos a realizar, intervenciones pedagógicas y docentes. También tiene lugar el diseño de proyectos áulicos, el esbozo de ideas para trabajar temáticas importantes para la comunidad y estrategias de sostenimiento de trayectorias individuales y grupales necesarias y oportunas que se puedan requerir. En estos espacios sería relevante la presencia de un profesor de inglés como partícipe de las discusiones que tienen que ver con la vida de la escuela y de las aulas, dialogando en torno a los criterios que se consensuen institucionalmente.

La carga horaria y presencialidad de los docentes en la escuela también podría optimizarse en función de rediseñar estrategias áulicas que contemplen el trabajo con las heterogeneidades de pensamiento, los estilos de aprendizajes de cada estudiante, sus saberes. Disponer y ofrecer las condiciones, herramientas y recursos que permitan a todos los alumnos apropiarse y manejar conocimientos. La cuestión clave, indudablemente, es ahondar en el descubrimiento de las diferencias de cada alumno para poder ofrecer una amplia variedad de estrategias, donde maestro y profesor de inglés acuerden y diseñen estrategias en forma conjunta para beneficiar el trabajo con los diferentes estilos de aprendizajes.

El trabajo colectivo también podría habilitar la discusión, inclusión y construcción de dinámicas en el aula que estén relacionadas con el trabajo de taller, la interacción grupal y la construcción social de conocimientos en el grupo-clase; la dinámica de taller como oportunidad de producción lingüística por su valor en cuanto a la construcción de objetos lingüísticos: búsqueda, lectura, audición y producción de discursos, exponentes de tipologías discusivas: textos escritos 
y orales como prácticas sociales. La dinámica de taller como oportunidad para que maestros y alumnos establezcan comunicación y construyan condiciones didácticas que den lugar a proyectos comunicativos: antologías, informes, publicaciones.

En el segundo grupo incluyo unas perspectivas sobre la enseñanza del inglés que, amalgamadas a estas condiciones institucionales que marcan las prácticas escolares, presentadas anteriormente, sin dudas redundarían en valiosos procesos de construcción de saberes y prácticas socialmente productivas en la escuela.

Se trata de configuraciones inéditas vinculadas con una perspectiva didáctica para la enseñanza del inglés, que piensa el trabajo crítico en la clase de inglés en diálogo con el contexto y que apuesta a la construcción de ciudadanías de estos tiempos, que advierte mirar lo local sosteniendo la mirada en lo global y encuentra sentido al trabajo con la lengua extranjera sólo anclándola en la comunidad donde viven nuestras niños. Me refiero a la puesta en valor de una perspectiva crítica que no sostenga la enseñanza de una lengua extranjera desde el vacío cultural (Barboni y Porto, 2011) sino que brinde oportunidades para la construcción de identidad hoy.

En tal sentido, apuesto a un necesario abordaje pedagógico y didáctico común desde ambas lenguas: la lengua materna y la lengua extranjera. Hablar de la enseñanza de las lenguas implica discutir diferentes teorías sobre la adquisición, lo que supone reconocer que el aprendizaje de una lengua implica considerar la cosmovisión que ésta sustenta. Destaco la importancia que tiene este proceso alfabetizador en las infancias que estamos abordando en este trabajo, las que están altamente surcadas por cuestiones sociales y económicas que atraviesan el trabajo áulico.

Desde este enfoque se propicia el desarrollo de competencias interculturales. Barboni (2011) sostiene que esta competencia intercultural se construye en el aula de inglés a través de las oportunidades de interacción con otros sujetos, otras culturas. Propone un espacio en que se desarrollen estrategias que posibiliten un encuentro cultural donde se pueda enriquecer el universo de los niños con culturas en las que se reconozcan puntos en común y donde también se establezcan diferencias conscientes. 
Hablar de una clase de inglés que aborde una perspectiva crítica y contextualizada socialmente implica un docente comprometido con el empoderamiento lingüístico y cultural de las infancias con las que trabaja, con el reconocimiento de estos niños como sujetos de derecho. El aula de lengua extranjera propiciando una suerte de anclaje entre culturas y tiempos, relacionando movimientos, personajes, situaciones que tienen relación con lo local. Hablo, también, del uso de la tecnología para explorar sitios y conocer perfiles de personalidades que no estén relacionadas con el éxito y la popularidad sin sentido, sino que hayan tenido que ver con lo que hoy nos pasa.

Se trata de reconocer al lenguaje como posibilidad de resignificación. A este respecto, la práctica docente se plantea, desde una perspectiva crítica, como práctica democrática en la que el trabajo con la lengua implica la construcción de un código que configure una práctica distinta, renovada y contrahegemónica, sosteniendo que a través del lenguaje se vehiculiza esta posibilidad de proponer una práctica alternativa que no sólo contemple, sino que intervenga. La idea es que los alumnos se pongan en contacto con discursos críticos en los que se generen discusiones sobre temas controversiales como la sexualidad, el poder, la desigualdad y su relación con el inglés, tratando de tomar distancia de un lugar imperialista. Una práctica docente que propicie aprendizajes críticos, donde los niños puedan utilizar los signos arbitrarios de esta lengua para resignificar sus propios significados, reestructurando el sentido en su comunidad.

Desde estas ideas, creemos que la construcción de la autonomía en la clase de inglés puede invitar a la construcción de un proyecto emancipador «Aquellos y aquellas que buscan la emancipación intentan conseguir el poder de controlar sus propias vidas en solidaridad con una comunidad orientada hacia la justicia» (Kincheloe et al., 2008:42). La clase de inglés, un espacio horizontal donde se pondrán en tensión las múltiples formas de dominación que perturban la autonomía y la toma de decisiones, a los que Barboni (2011) denomina procesos de americanización, europeización, y mcdonalización. Dispositivos de manipulación que las infancias consumen a través de la televisión, películas, Internet, y muchas otras formas artísticas y culturales. Construir, hacer visibles los saberes socialmente productivos, en este contexto, es un trabajo político y pedagógico que sólo se puede llevar a cabo en el marco de un proyecto pedagógico colectivo. Claramente, esta perspectiva nos invita a descubrir los 
temas que se tensionan entre la alienación y la preocupación en el seno de las comunidades pobres para organizar proyectos democráticos. El docente debe reconocer aquellos temas que signifiquen un problema para cada comunidad, ponerlos en discusión en la clase a través de diferentes portadores: textos breves, titulares, frases, canciones, poemas, palabras claves. En definitiva, se trata de propiciar, también en la clase de inglés, la desnaturalización de temas, discutir las condiciones en que se generan en cada cultura, y favorecer la concientización de las diferencias en cada lugar.

La enseñanza del inglés en la escuela primaria plantea desafíos para pensar una didáctica específica en contextos de pobreza. Los márgenes de la ciudad de Santa Fe están cargados de problemas actuales y complejos. Caminar los bordes de esta ciudad implica encontrarse con vicisitudes propias de cada comunidad que pertenecen a grandes problemas transversales que, sin dudas, habilitan lugares para la conexión entre el aula y otras culturas.

Una práctica de la enseñanza de lengua extranjera que se constituya desde este territorio simbólico y material que configura al lenguaje, propiciando un diálogo plurilingüe con el contexto y, también, con otros lenguajes, como el de las ciencias, abre la maravillosa oportunidad de trabajar en forma contextualizada, abandonar historias «ideales» de personajes inventados para dar lugar a temas reales donde lo lingüístico esté ampliamente relacionado con lo social, a tal punto de ser la oportunidad de problematizar lo cotidiano, incluso en forma conjunta con otras asignaturas.

La idea de visibilizar, desde la enseñanza de una lengua extranjera, a las ciudadanías de los bordes santafesinos es una apuesta a la posibilidad que puedan interrogar su propia realidad, problematizar lo cotidiano, apresar un otro presente en el que quepamos todos. 


\section{Referencias bibliográficas}

Achilli, E. (1990). Antropología e investigación educacional. Aproximación a un enfoque constructivista indiciario. III Congreso Argentino de Antropología Social. Rosario, Argentina.

Barboni, S. y Porto, M. (Eds.) (2011). Enseñanza de Inglés e Identidad Nacional. A los 200 años de la Revolución de Mayo. La Plata: Ediciones al Margen.

Brailovsky, D. (2008). Sentidos Perdidos de la Experiencia Escolar. Angustia, desazón, reflexiones. Colección Ensayos y Experiencias № 70. Buenos Aires: Novedades Educativas.

Carr, W. y Kemmis, S. (1988). Teoría Crítica de la Enseñanza. La investigación - Acción en la formación del Profesorado. España: E. Martínez Roca.

Duschatzky, S. (2008). La escuela como frontera: Reflexiones sobre la experiencia escolar de jóvenes de sectores populares. Buenos Aires: Paidós.

Edelstein, G. y Coria, A. (1995). Imágenes e imaginación. Iniciación a la Docencia. Buenos Aires: Kapelusz. Giroux, H. (1997). Pedagogía y política de la esperanza. Teoría, cultura y esperanza. Buenos Aire -Madrid: Amorrortu.

Gómez Sollano, M. (2009). Saberes Socialmente Productivos y Educación. Contribuciones al Debate. Serie: Macroproyecto Ciencias Sociales y Humanidades. México: Universidad Nacional Autónoma de México.

Gutiérrez, F. (2010). A vueltas con la globalización del inglés: expectativas y paradojas. Historia y Comunicación Social, (15), 27-45. Universidad Complutense de Madrid.

Klett, E. (2013). Didáctica de las Lenguas Extranjeras. Tras un desarrollo disciplinar autónomo. Didáctica de las Lenguas Extranjeras, Universidad Nacional de Luján, Buenos Aires. Recuperado de http:// www. didacticale.unlu.edu.ar/?q=node/17 (consultado el 06/03/15).
Kincheloe, J. y McLaren, P. (2008). Pedagogía Crítica. De qué hablamos, dónde estamos. Barcelona: Crítica y Fundamentos.

Kumaravadivelu, B. (1994). The Postmethod Condition: (E)merging Strategies for Second/Foreign Language Teaching. TESOL Quarterly, Vol. 28(1, Spring), 27-48. Recuperado de http://links.jstor.org/sici?sici $=0039-8322 \% 28199421 \% 2928 \% 3 \mathrm{~A} 1 \% 3 \mathrm{C} 27 \%$ 3ATPC\%28SF\%3E2.0.CO\%3B2-2TESOL

(2001). Toward a Postmethod Pedagogy. TESOL Quarterly Vol. 35(4, Winter), 537-560. Recuperado de http://www.bkumaravadivelu.com/articles\%20in\%20pdfs/2001\%20Kumaravadivelu\%20 Postmethod\%20Pedagogy.pdf

(2003). Macrostrategies for Language Teaching. Yale: University Press.

Manga, A. (2008). Lengua Segunda (L2) Lengua Extranjera (LE): Factores e Incidencias de Enseñanza/Aprendizaje. Tonos, (16). Recuperado de http:// www.um.es/tonosdigital/znum 16/secciones/estudios--10-Ensenanza.htm

McLaren, P. y others (2008). Pedagogía Crítica. De qué hablamos, dónde estamos. Barcelona: Graó, Crítica y Fundamentos.

Ministerio de Educación, Ciencia y Tecnología de la Nación Argentina (2006). Ley de Educación Nacional 26206

Puiggrós, A.; Visacovsky, A. et al. (2004). La fábrica del Conocimiento. Los saberes socialmente productivos en América Latina. Rosario: Homo Sapiens.

Sagastizábal, M.Á. (Coord.) (2006). Aprender y enseñar en contextos complejos. Multiculturalidad, diversidad y fragmentación. Buenos Aires: Novedades Educativas.

Sautu, R. (2003). Todo es teoría. Objetivos y métodos de investigación. Buenos Aires: Lumiere. 
Sirvent, M. (1996). Múltiples pobrezas. Conferencia. Panel de Apertura. Congreso Internacional de Educación. Buenos Aires, Facultad de Filosofía y Letras Universidad Nacional de Buenos Aires, 24, 25 y 26 de julio. Recuperado de http://www.webiigg.sociales.uba. ar/conflictosocial/libros/violencia/12_SIRVENT,\%20 Multipobrezas,\%20Violencia\%20y\%20Educacion.pdf Tusón Valls, A. (2002). El análisis de la conversación: entre la estructura y el sentido. Estudios de Sociolingüística, 3(1), 2002, 133-153. Recuperado de https://www.textosenlinea.com.ar/academicos/El\%20 an\%C3\%A1liis\%20de\%20la\%20conversacion.pdf

Fecha de recepción: 01/07/18

Fecha de aceptación: 10/10/18 